\title{
Lifetime statistics in chaotic dielectric microresonators
}

\author{
Henning Schomerus \\ Department of Physics, Lancaster University, Lancaster LA1 4YB, United Kingdom \\ Jan Wiersig \\ Institut für Theoretische Physik, Universität Magdeburg, Postfach 4120, D-39016 Magdeburg, Germany
}

Jörg Main

1. Institut für Theoretische Physik, Universität Stuttgart, 70550 Stuttgart, Germany

(Dated: April 16, 2009)

\begin{abstract}
We discuss the statistical properties of lifetimes of electromagnetic quasibound states in dielectric microresonators with fully chaotic ray dynamics. Using the example of a resonator of stadium geometry, we find that a recently proposed random-matrix model very well describes the lifetime statistics of long-lived resonances, provided that two effective parameters are appropriately renormalized. This renormalization is linked to the formation of short-lived resonances, a mechanism also known from the fractal Weyl law and the resonance trapping phenomenon.
\end{abstract}

PACS numbers: 42.55.Sa, 42.25.-p, 42.60.Da, 05.45.Mt

\section{INTRODUCTION}

Over the past decade optical microresonators have attracted considerable attention in various fields of physics [1]. Microresonators are basic components for single-photon emitters [2], ultralow threshold lasers [3, 4], and solid-state cavity quantum electrodynamics [5-7], to name just a few of the possible applications. Deformed dielectric microdisk resonators are of particular interest since they allow for directional light emission, an effect that can be understood and optimized by exploring fundamental principles of ray-wave correspondence [8-13]. This directionality can be employed to design microlasers which make best use of the total output intensity. Whether a carefully designed mode admits substantial lasing depends on how its lifetime compares to the lifetimes of other modes in the amplification window. This information is encoded in the imaginary parts of the resonance frequencies. However, the associated spectral statistics have been studied only for a few special geometries, such as the circular disk without and with surface roughness [14, 15], while other investigations focused on disordered media [16, 17] and resonators with small openings [18-24].

In order to address the spectral properties of dielectric microdisk resonators from a broader perspective it is desirable to adopt concepts which have been successfully applied to other classes of open systems. In particular, the characterization of spectral statistics is often based on two ingredients: (a) A Weyl law [25], which estimates the number of states within a given frequency interval, and (b) random-matrix theory (RMT) [26], which addresses the statistical fluctuations and correlations of these states. Traditionally both concepts have been applied to systems with small openings, for which the Weyl law is identical to that of the closed counterpart (where the number of states is estimated by assigning one state per Planck cell in phase space), while RMT can be justified because modes of the closed system become strongly mixed on the scale of the typical life time of the quasibound states in the open system.
A framework how to adapt these concepts to dielectric microdisk resonators, which are relatively more open systems allowing for refractive escape along the entire boundary, has only emerged very recently. Due to the short typical lifetime, strong mode mixing is only observed for a reduced number of quasibound states. For systems with ballistic escape, where the mode-mixing time scale is given by the Ehrenfest time [27-30] and the support of the long-living states is the fractal repeller, it has been established that the number of quasibound states that exceed a certain lifetime is given by a fractal Weyl law [31-35]. Based on a generalization of the repeller for refractive escape, the significance of the fractal Weyl law to dielectric microdisk systems with small refractive index has been demonstrated for the stadium geometry in Ref. [36]. In the present work we consider the same geometry and explore the predictive power of RMT, whose extension to dielectric microresonators was proposed in Ref. [37]. The application of this extension requires to determine two effective parameters (a time scale for the internal dynamics and an effective number of states), which are sensitive to the formation of short living resonances (a mechanism also at heart of the fractal Weyl law [33], as well as the resonance trapping phenomenon [38]). We concentrate on moderately large values of the refractive index, for which the short-living resonance states resemble the bouncing-ball modes of the closed stadium billiard [39], and find that the numerical lifetime statistics of long-living quasibound states agree very well with the RMT predictions.

The outline of this paper is as follows. The background Section II provides a brief introduction into the spectral properties of dielectric microdisk resonators and the dynamical model of Ref. [37]. In Section III we present numerical results for complex resonance frequencies in stadium-shaped microresonators and then analyze the statistics of long-living resonances in two steps. First we show that the mean properties of the resonance frequencies can be characterized in terms of the two effective parameters mentioned above. Based on these parameters, we secondly show that fluctuations of the life times are well captured by RMT. The results are summa- 
rized in the concluding Sec. IV.

\section{THEORETICAL BACKGROUND}

\section{A. Dielectric microdisk resonators}

Dielectric microdisk resonators effectively have a 2D geometry, defined by a region of constant refractive index $n$ which is embedded into vacuum (where the refractive index is unity). The classical dynamics inside such a resonator is similar to a billiard, where a point-like particle moves freely in a two-dimensional domain with elastic reflections at the boundary [40]. Depending on the shape of the boundary a billiard can show a variety of dynamical behaviors ranging from integrable to fully chaotic [41]. Light rays in a microdisk behave similarly as they are totally reflected at the interfaces as long as the angle of incidence is larger than the critical angle for total internal reflection. If, however, the angle of incidence is smaller than the critical angle, light can escape refractively according to Snell's and Fresnel's laws. Hence, dielectric microdisk resonators are leaking billiards $[8,9,42]$.

The leakiness of the resonator limits the photon lifetime, which can be described by associating complex values to the resonance frequencies $\omega$. These frequencies can be obtained from the Helmholtz equation [43]

$$
-\nabla^{2} \psi=n^{2}(x, y) \frac{\omega^{2}}{c^{2}} \psi
$$

where $c$ is the speed of light in vacuum. For a piecewise constant refractive index, this equation holds for both transverse magnetic (TM) and transverse electric (TE) polarization. For TM polarization the electric field is perpendicular to the resonator plane with $E_{z}=\operatorname{Re}\left[\psi(x, y) e^{-i \omega t}\right]$, and the wave function $\psi$ and its normal derivative $\partial_{\perp} \psi$ are continuous across the boundary of the resonator. For TE polarization, $\psi$ represents the $z$-component $H_{z}$ of the magnetic field, and the functions $\psi$ and $n^{-2} \partial_{\perp} \psi$ are continuous across the boundary. For plane interfaces, these boundary conditions yield the Fresnel reflection amplitudes

$$
\begin{aligned}
& r_{\mathrm{TM}}(p)=\frac{\sqrt{1-p^{2}}-\sqrt{n^{-2}-p^{2}}}{\sqrt{1-p^{2}}+\sqrt{n^{-2}-p^{2}}}, \\
& r_{\mathrm{TE}}(p)=\frac{\sqrt{1-p^{2}}-n \sqrt{1-n^{2} p^{2}}}{\sqrt{1-p^{2}}+n \sqrt{1-n^{2} p^{2}}}
\end{aligned}
$$

for resonator photons with dimensionless transverse momentum $p=\sin \chi$, where $\chi$ is the angle of incidence.

At infinity, outgoing wave conditions are imposed which results in quasi-bound states with frequencies $\omega$ situated in the lower half of the complex plane. Whereas the real part is the usual frequency, the imaginary part is related to the lifetime $\tau=-1 /[2 \operatorname{Im} \omega]$. The quality factor of a quasi-bound state is defined by $Q=-\operatorname{Re} \omega /[2 \operatorname{Im} \omega]$.

An alternative method to obtain the quasibound states sets out with the scattering matrix $S$, which relates the amplitudes of the incoming wave components to the amplitudes of the outgoing wave components (see, e.g., Refs. [18, 19, 21-23]). At real frequencies, the scattering matrix is unitary. The condition of a quasibound state corresponds to the situation where outgoing wave components are admitted in absence of any incoming radiation. Under these conditions the scattering matrix diverges. The resonance frequencies therefore correspond to the poles of the scattering matrix in the complex frequency plane.

\section{B. Dynamical model of microresonators}

Random-matrix theory delivers a statistical description of complex quantum systems on the basis of just a few system parameters. In particular, a classification according to fundamental symmetries such as time-reversal symmetry or spinrotation symmetry allows to identify ten classes, among which three are the traditional Wigner-Dyson classes [26], while a further seven classes apply to systems with chiral or particlehole symmetries [44]. For each symmetry class one can consider two basis variants of RMT, which either concerns hermitian matrices $H$ (representing the Hamiltonian) or unitary matrices $U$ (representing the scattering matrix or the time evolution operator). In the absence of magneto-optical effects photonic systems are represented by the orthogonal symmetry class, in which the hermitian and unitary matrices are symmetric under transposition $\left(H=H^{T}, U=U^{T}\right)$.

Traditional random-matrix theory does not assume any further knowledge of the system, which leads to a uniquely defined invariant probability measure (the Haar measure). Physical systems, however, often contain additional commonalities. Dielectric microresonators are a good example, since the escape is governed by well structured Fresnel laws of reflection and refraction. These laws not only affect the far-field radiation characteristics, but also determine the confinement, and therefore impact on the spectral properties. It is therefore desirable to incorporate the specific confinement characteristics of dielectric microresonators into RMT. In order to study spectral statistics, one furthermore needs to accommodate frequency correlations associated to the internal dynamics in the resonator. Both requirements are met by a variant of RMT recently proposed in Ref. [37]. In this model the scattering matrix takes the form

$$
S=-\mathcal{R}+\mathcal{T} U[\exp (-i \omega \tau)-\mathcal{R} U]^{-1} \mathcal{T}
$$

where the diagonal matrices

$$
\mathcal{R}_{l m}=\delta_{l m} r\left(p_{l}\right), \quad \mathcal{T}_{l m}=\delta_{l m} \sqrt{1-\left|r\left(p_{l}\right)\right|^{2}}
$$

are determined from the Fresnel reflection amplitudes (2) at discrete impact parameter $p_{l}=(2 l-M-1) / M$ (with $M=\operatorname{dim} \mathrm{S}$ and $l=1, \ldots, M)$ [45]. The frequency dependence is characterized by a time scale $\tau$ which represents the typical time of flight through the resonator. The $M$ dimensional matrix $U$ can be interpreted as a unitary time evolution operator which propagates photons between encounters with the interface. 


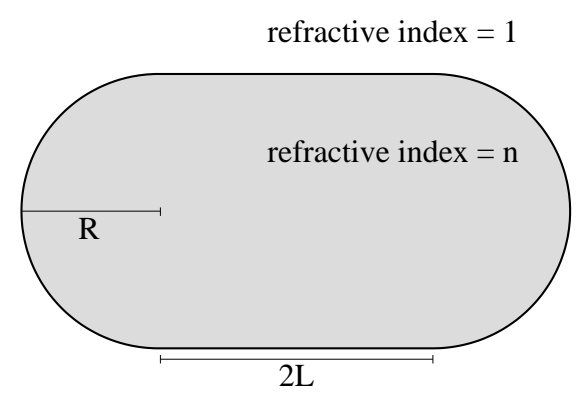

FIG. 1: Sketch of a stadium-shaped dielectric microresonator with $L=R$.

In the dynamical model (3), the poles of the scattering matrix are determined by the eigenvalue equation

$$
\mathcal{R} U \psi=\exp (-i \theta) \psi
$$

where $\theta=\omega \tau$ is a dimensionless quantity. The operator on the left is subunitary, and therefore each resonance frequency in general possesses a negative imaginary part related to the lifetime of the state. Eigenvalue problems of this type with various specifications of $\mathcal{R}$ have recently attracted considerable attention [33-35, 46-49], with main focus on systems with small or ballistic openings (as represented by quantum dots with quantum point contacts).

In the RMT version of the dynamical model (3), $U$ is taken from the circular orthogonal ensembles. The statistical description is then completed by the specification of the two parameters $M$ and $\tau$. Once these parameters are determined, one can investigate statistical fluctuations. We now implement this strategy for a specific model system.

\section{LIFE TIME STATISTICS IN THE STADIUM MICRORESONATOR}

A microdisk resonator with stadium-shaped cross-section is illustrated in Fig. 1. The region with refractive index $n$ is bounded by two semicircles with radius $R$ and two straight lines of length $L$. The corresponding closed cavity, the stadium billiard, is a paradigm for fully developed chaos [50, 51]. We consider the case $L=R$, for which the closed classical billiard has the largest Lyapunov exponent [52].

The open stadium resonator has been studied both theoretically and experimentally in a number of works, with focus on the spatial mode structure and ray-wave correspondence [5357]. In a first step towards the characterization of the resonance frequency spectrum of the stadium resonator, the applicability of the Weyl law has been investigated in Ref. [36].

We start our statistical analysis of the spectral fluctuations with the numerical determination the resonance frequencies. In the next step, we relate the averages of the real and imaginary parts of these frequencies to the two parameters $\tau$ and $M$ of the dynamical model (3). This fixes the RMT version of this model and delivers a prediction for the distribution of life times, which we compare in the final step to the actual life time statistics of the stadium resonator.

\section{A. Resonance frequencies}

The resonance states in the stadium billiard can be classified by their parity with respect to the horizontal and vertical symmetry lines of the stadium billiard. In the computations this can be exploited by considering four desymmetrized versions of the resonator, which differ by the boundary conditions imposed on the two symmetry lines (Dirichlet or Neumann boundary conditions for states of odd or even parity with respect to the given symmetry line, respectively). As explained in detail in Ref. [36] the boundary element method [58] and the harmonic inversion technique [59] are very efficient tools to compute the quasibound states. The combination of these two methods allows us to gather a large number of resonant frequencies $\omega$, as is required for a reliable statistical analysis. A dimensionless form of these frequencies can be obtained by considering the scaled frequencies $\Omega=\omega R / c$. For the present study we collected resonance frequencies in the interval $66<n \operatorname{Re} \Omega<82.5$ for both polarizations (TM and TE), all parities, and two different values of the refractive index, $n=3.3$ (for GaAs) and $n=5$. The latter value we have chosen since a broad interval of refractive indices allows a more convincing validation of our theory.

For illustration, the position of the dimensionless resonance frequencies in the complex plane for $n=5$ and TM polarization (all parities) is shown in Fig. 2. The figure shows a clear separation of short-living resonances which are organized along well-structured bands ( $\operatorname{Im} \Omega \lesssim-0.015)$, and long living resonances whose position does not suggest any clear organization principle $(\operatorname{Im} \Omega \gtrsim-0.015)$, and therefore call for a statistical analysis.

Figure 3(a) and (b) shows examples of long-lived and shortlived quasibound states in the chaotic microstadium. The complex spatial pattern of the long-lived state is in strong contrast to the rather simple pattern of the short-lived state. Shortlived states in the microstadium resemble bouncing-ball states in the closed stadium billiard [39], which can be characterized by two quantum numbers corresponding to the number of nodal lines in horizontal and vertical direction. Bouncingball states are usually ignored in the statistical analysis of the quantum spectrum of closed systems [60]. In the same spirit we restrict in the following our attention to the long-living resonances, which are selected via a cut-off of the decay rate $(\operatorname{Im} \Omega>-0.04$ for $n=3.3, \mathrm{TM} ; \operatorname{Im} \Omega>-0.1$ for $n=3.3$, TE; $\operatorname{Im} \Omega>-0.015$ for $n=5$, TM; $\operatorname{Im} \Omega>-0.025$ for $n=5$, TE; in all cases we observe the same clear separation of long- and short living states as in Fig. 2).

\section{B. Determination of characteristic parameters}

A useful reference point for the quantification of the spectral fluctuations of the long-living resonances are the frequency and time scales set by their mean spacing along the real frequency axis, and their mean decay rate. The numerical values of the dimensionless quantities $(\Delta \Omega)_{\mathrm{s}}^{(\text {tot })}$ and $-\langle\operatorname{Im} \Omega\rangle_{\mathrm{s}}$ are collected in Tab. I. Here the subscript 's' refers to the stadium billiard, while the superscript 'tot' for the spac- 

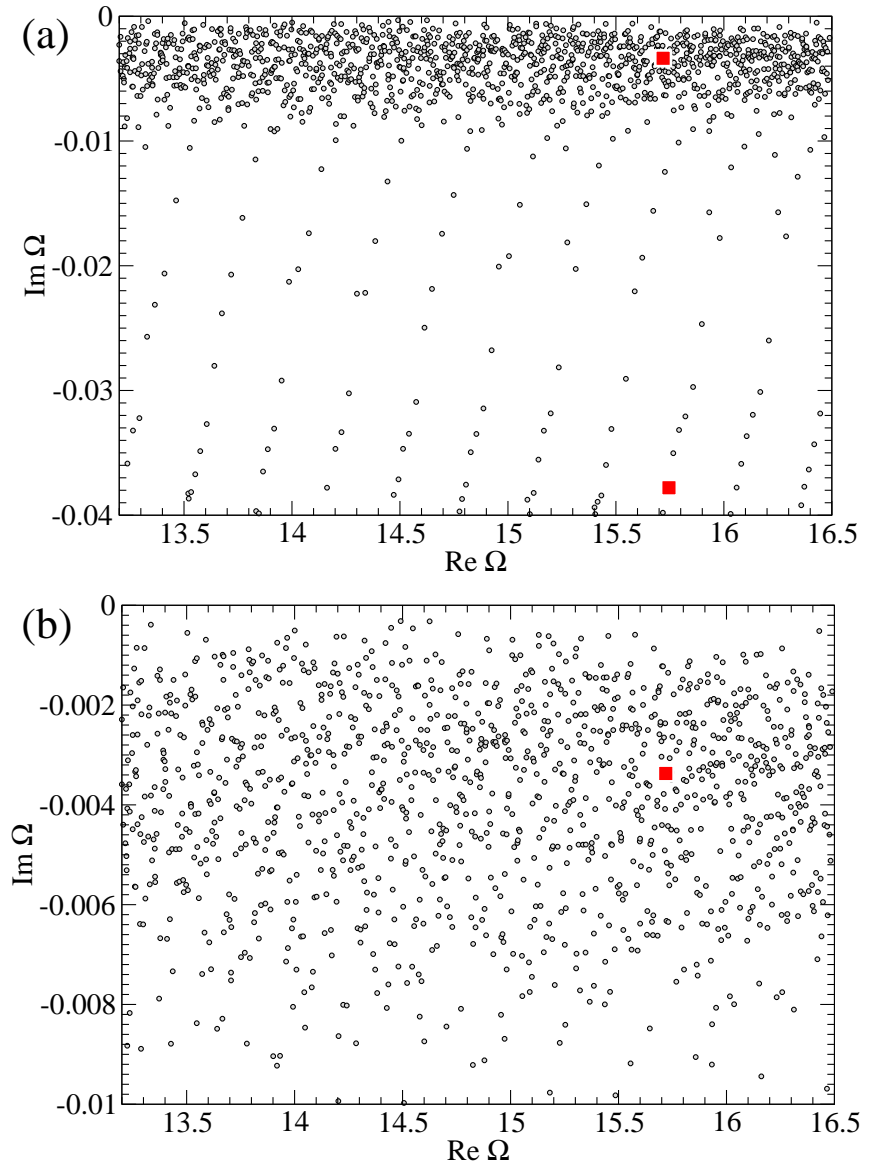

FIG. 2: (Color online). (a) Position of dimensionless complex resonance frequencies with TM polarization for the stadium microresonator with refractive index $n=5$. The filled squares indicate the quasibound states shown in Fig. 3. (b) Same but zoomed in onto the long-living resonance frequencies.

ing indicates that we do not discriminate the resonances by parity. We now describe how this information can be used to determine the parameters $M$ and $\tau$ which serve as input of the RMT model. Because of parity conservation, we are interested in the effective parameters per symmetry class; the total effective matrix dimension irrespective of parity is denoted by $M^{\text {(tot) }}=4 M$.

Let us first assume that we could ignore the existence of well organized sequences of short-living resonances with nonchaotic wave patterns, i.e., the case of a fully wave-chaotic resonator. The mean resonance spacing then follows the ordinary Weyl law (one state per Planck cell in phase space [25]),

$$
(\Delta \Omega)_{\text {Weyl }}^{(\text {tot })}=\frac{2 \pi}{n^{2} \operatorname{Re} \Omega} \frac{R^{2}}{A},
$$

where $A=(4+\pi) R^{2}$ is the area of the billiard. Analogously, the dimension $M^{\text {(tot) }}$ of the scattering matrix can be estimated by applying the Weyl law to the perimeter $S=(2 \pi+4) R$ of the billiard (one channel per half wave length),

$$
M_{\mathrm{Weyl}}^{(\mathrm{tot})}=\frac{n \operatorname{Re} \Omega}{\pi} \frac{S}{R} .
$$

(a)

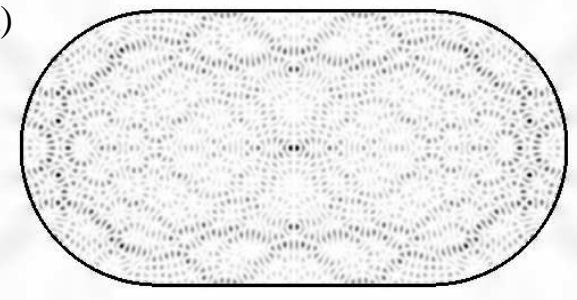

(b)

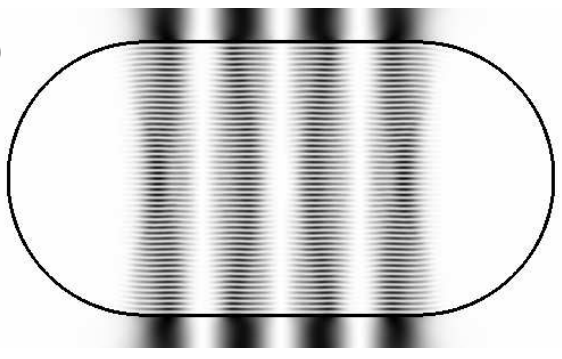

FIG. 3: Near-field pattern of a long-lived (a) and a short-lived quasibound states (b) with $\Omega=15.71886-i 0.00337$ and $\Omega=$ 15.74567 - i0.0378. As in Fig. 2, these states are computed for TM polarization and $n=5$.

TABLE I: The first two rows compare the dimensionless mean frequency spacing of long-living resonances in the stadium resonator in the interval $66<\operatorname{Re}(n \Omega)<82.5$ (irrespective of parity) to the prediction from the Weyl law (5). The third and fourth row tabulate the mean decay rate of the long-living resonances and the mean dimensionless decay rate in the dynamical model (3) (obtained in RMT in the asymptotic limit $M \gg 1$ ). As described in the text, the relative scaling of these quantities can be used to determine the time scale $\tau$ [Eq. (9)] and effective matrix dimension $M=M^{\text {(tot) }} / 4$ (for fixed parity) [Eq. (10)], which are tabulated in the last two rows.

\begin{tabular}{|l|c|c|c|c|}
\hline \hline & $n=3.3, \mathrm{TM}$ & $n=3.3, \mathrm{TE}$ & $n=5, \mathrm{TM}$ & $n=5, \mathrm{TE}$ \\
\hline$(\Delta \Omega)_{\mathrm{s}}^{(\mathrm{tot})}$ & 0.00410 & 0.00418 & 0.00259 & 0.00263 \\
$(\Delta \Omega)_{\mathrm{Weyl}}^{(\mathrm{tot})}$ & 0.00359 & 0.00359 & 0.00237 & 0.00237 \\
\hline$-\langle\operatorname{Im} \Omega\rangle_{\mathrm{s}}$ & 0.0143 & 0.0272 & 0.00395 & 0.00736 \\
$-\langle\operatorname{Im} \theta\rangle_{\mathrm{RMT}}$ & 0.150 & 0.268 & 0.0645 & 0.1137 \\
\hline$\tau / \tau_{0}$ & 1.45 & 1.37 & 1.50 & 1.42 \\
$M$ & 37 & 38 & 37 & 38 \\
\hline \hline
\end{tabular}

In the dynamical model, eigenvalue equation (4) has $M$ solutions with uniform statistical distribution of the phase $\operatorname{Re} \theta$. This translates into a mean dimensionless frequency spacing

$$
(\Delta \Omega)^{(\text {tot })}=\frac{2 \pi}{M^{(\text {tot })}} \frac{R}{\tau c} .
$$

The combination of Eqs. (5), (6), and (7) results in the relation

$$
\tau=\frac{n \pi A}{c S} \equiv \tau_{0}
$$

which coincides with Sabine's law for the mean propagation time between encounters with the boundary. If applied to resonances of a fixed parity, the frequency spacing $\Delta \Omega=$ $4(\Delta \Omega)^{\text {(tot) }}$ quadruples, while the effective matrix dimension 
$M=M^{(\text {tot })} / 4$ is reduced by a factor four. It follows from Eq.

(7) that the time scale $\tau$ is not affected by these substitutions.

The finiteness of the time to establish wave chaos in an open system results in the reduction of the number of longliving quasibound states with respect to the prediction of the ordinary Weyl law [Eq. (5)]; this is compensated by the formation of short-lived states [33]. A striking example is the emergence of a fractal Weyl law for systems with an Ehrenfest time which is comparable or larger than the typical life time [31-35]. The formation of short-lived states also often results in resonance trapping, a phenomenon which reduces the average decay rate of the long-living resonances (as dictated by sum rules for the total coupling strength of the system to the exterior; see Ref. [38] for a detailed discussion). The existence of well-organized bands of resonances deep in the complex-frequency plane (see Fig. 2(a)) indicates that both mechanisms are at work in the stadium resonator. As we now show, the consequences can be described by renormalized parameters $M$ and $\tau$.

Since the reduced number of long-living resonances and resonance trapping both impact on the average characteristics of complex frequencies (affecting the real and imaginary part, respectively), the renormalized parameters can be determined without resorting to the statistical fluctuations. As a first relation, we compare the mean decay rate of long-living states in the stadium to the prediction of RMT,

$$
\frac{c \tau}{R}=\frac{\langle\operatorname{Im} \theta\rangle_{\mathrm{RMT}}}{\langle\operatorname{Im} \Omega\rangle_{\mathrm{s}}}
$$

For the second relation we evaluate Eq. (7) using the numerically observed frequency spacing of the long-living resonances, $M_{\mathrm{s}}^{(\mathrm{tot})}=\left[2 \pi /(\Delta \Omega)_{\mathrm{s}}^{(\mathrm{tot})}\right][R / c \tau]$. The effective matrix dimension per symmetry class of fixed parity is therefore given by

$$
M=\frac{\pi}{2(\Delta \Omega)_{\mathrm{s}}^{(\text {tot })}} \frac{R}{c \tau} .
$$

In principle, Eqs. (9) and (10) need to be solved selfconsistently because the RMT average $\langle\operatorname{Im} \theta\rangle_{\mathrm{RMT}}$ also depends on $M$. For large values of $M$, however, the RMT average saturates (see insets of Fig. 6). Practically, therefore, $\tau$ can be obtained from Eq. (9) by fixing $M$ to a large value. The actual value of $M$ then follows from (10). This procedure is consistent if the resulting value $M \gg 1$. Table I tabulates the values for $\tau$ and $M$ which result when this procedure is applied to the resonances of the stadium resonator.

For all four combinations of refractive index and polarization, we find that the collision time $\tau$ differs from the prediction $\tau_{0}$ of a totally wave-chaotic resonator by a factor of order unity, while the effective matrix dimension $M$ is also almost identical in all cases. Taking the mean over all four cases we obtain the values

$$
\tau=1.44 \tau_{0}, \quad M=38 .
$$

The relation $\tau>\tau_{0}$ can be attributed to the abovementioned resonance-trapping mechanism, according to which the
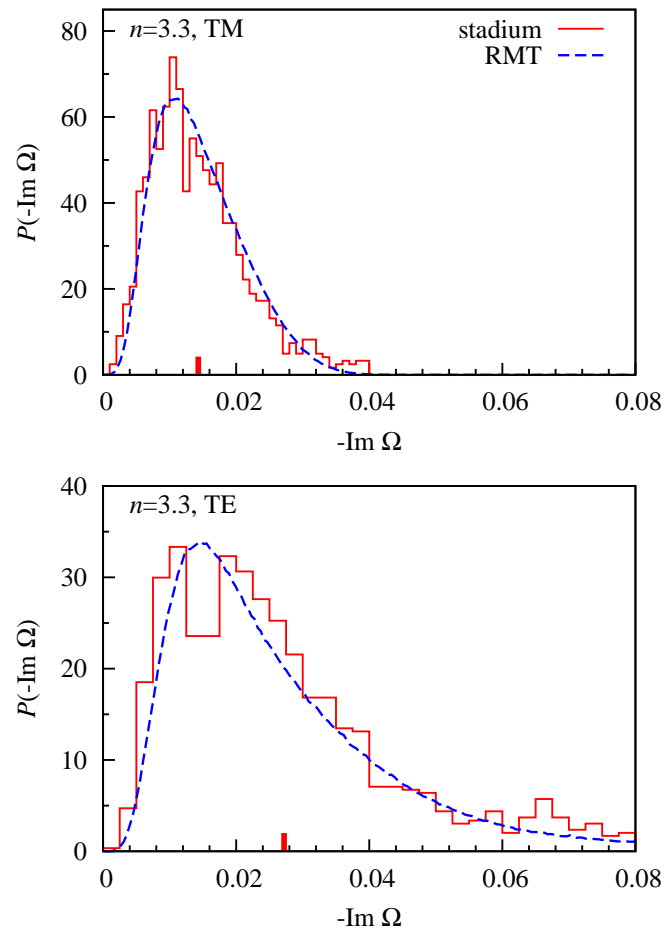

FIG. 4: (Color online). Distribution of decay rates (in dimensionless units) for resonances in the stadium resonator with refractive index $n=3.3$ (histogram), compared to the prediction of RMT (dashed curve) based on the dynamical model (3) with $\tau=1.44 \tau_{0}$ and $M=$ 38. Short-living resonances in the stadium which form bands in the complex- $\Omega$ plane (see Fig. 2) are neglected via a cut-off. Top panel: TM polarization, $\operatorname{Im} \Omega>-0.04$. Bottom panel: TE polarization, $\operatorname{Im} \Omega>-0.1$. The large ticks indicate the value of the average dimensionless decay rate (see Tab. I).

strong coupling of the short-living states to the exterior of the resonator must be compensated by a reduced coupling of the long-living states [38]. The constancy of $M$ and $\tau$ over all four combinations can be attributed to the fact that we compare equivalent frequency intervals $66<n \operatorname{Re} \Omega<82.5$, while the internal classical ray dynamics is also fixed. Since the total number of resonance frequencies in all cases agrees well with the ordinary Weyl law (5), we can further conclude that in all four cases the fraction of short- and long-living states is comparable.

\section{Life time statistics}

When a resonator is employed as a microlaser, the quasibound states with the smallest decay rate within the amplification window tend to be the first that cross the lasing threshold. In a simple model, lasing occurs as soon as the gain counters the losses through the interfaces [16, 19, 21-23]. The analysis of the mean decay rate in the preceding section therefore delivers a rough estimate of the necessary gain at the lasing threshold. The distribution of resonances in the complex plane (Fig. 2), however, suggests that the decay rates fluctuate 

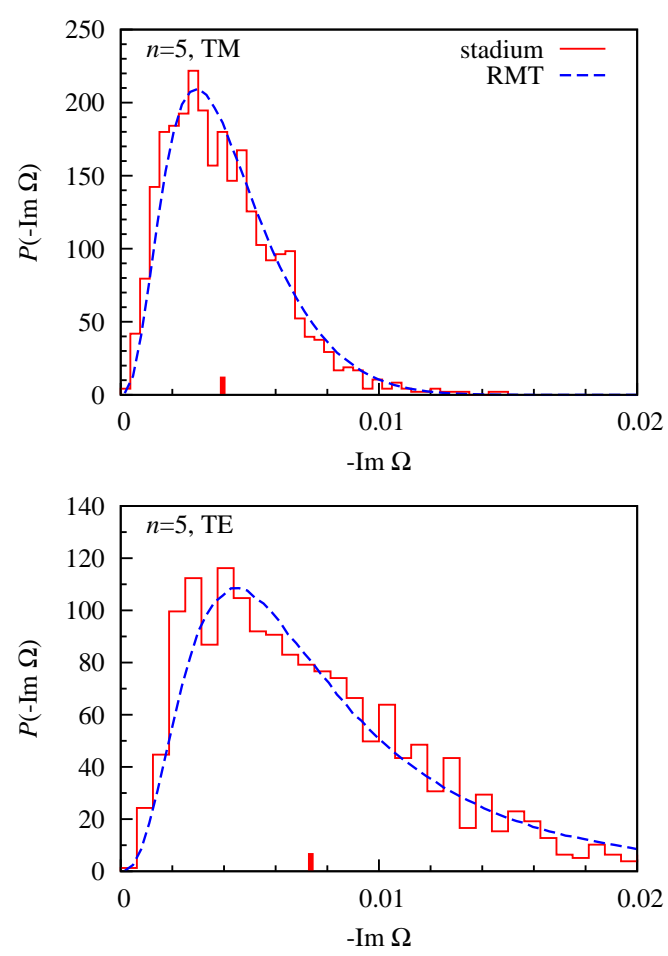

FIG. 5: (Color online). Same as Fig. 4, but for higher refractive index, $n=5$. The cut-offs are $\operatorname{Im} \Omega>-0.015(\mathrm{TM})$ and $\operatorname{Im} \Omega>$ -0.025 (TE).

broadly. Such large fluctuations entail that some states have a much smaller decay rate, which results in a reduced lasing threshold. We now turn to the question whether these fluctuations are captured by the RMT variant of the dynamical model (3).

Histograms of the decay rates of the resonances in the stadium resonators are shown in Figs. 4 and 5. For comparison, the figures also show the prediction of the RMT model, with parameters given by Eq. (11). Since these parameters apply to the quasibound states of a fixed parity, the total spectral statistics are obtained by the superposition of four systems with identical effective parameters, but independent microscopic realizations of the $M$-dimensional random matrix $U$. The RMT results in Figs. 4 and 5 are based on 50000 realizations per combination of polarization and refractive index.

In all cases we find excellent agreement of all key features, such as the modal value (with maximal probability) of the decay rate, the width of the peak around the modal value, and the shape of the distribution in the two tails. In particular, it should be noted that the average decay rates (tabulated in Tab. I, and shown as large ticks in the figures) are noticeably larger than the modal value of the decay-rate distribution. This difference arises from the long tail of resonances with relatively large decay rate, which closely follows a slow power law. Such power-law tails are a robust feature of RMT models [18], including models formed on the basis of the eigenvalue problem (4) [48]. As illustrated for $n=5$ in Fig. 6, in the RMT model the width of the distribution around the
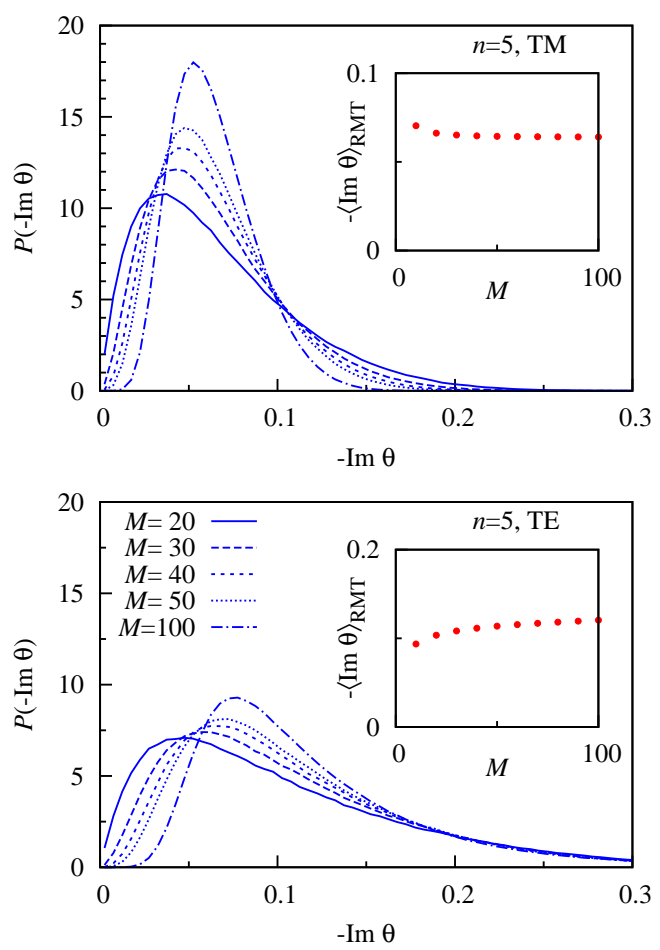

FIG. 6: (Color online). Comparison of lifetime distribution in the RMT model for various numbers of channels $M$, with $n=5.0$ (top panel: TM; bottom panel: TE). The insets show the $M$-dependence of the RMT average $\langle\operatorname{Im} \theta\rangle_{\mathrm{RMT}}$.

modal value depends noticeably on $M$. The good agreement of the width with the actual histograms in Figs. 4 and 5 lends direct support to the procedure employed in the determination of the renormalized effective parameters (11). We emphasis that the renormalized parameters are determined independently and do not result from a fitting to the numerically computed lifetime statistics.

\section{SUMMARY}

Dynamical models with scattering matrices of the form (3) have recently attracted considerable attention, both within RMT [46-48] as well as for individual dynamical systems. In particular, these models have been explored in association with the Ehrenfest time [28-30] and the related question of fractal Weyl laws [33-35, 49]. Some investigations of the Ehrenfest time employ an effective RMT model for the scattering matrix [61-63]. In these investigations it was found that certain transport properties (such as universal conductance fluctuations and shot noise) are faithfully described by effective RMT, while others (such as the weak-localization correction) are not [29].

The present work is motivated by the suggestion in Ref. [37] that the dynamical model (3) can also be used to describe the spectral fluctuations of long-living resonances in chaotic dielectric microresonators. We combined this model 
with a variant of effective RMT, which is directly applied to the internal round-trip operator $U$ [33].

We confirm that the RMT variant of the dynamical model captures the key features of the life time statistics in a specific dielectric microresonator, the stadium-shaped resonator. This comparison requires a careful determination of the effective collision time $\tau$ of photons with the interfaces and the effective number of modes $M$ that are mixed by the reflections at the boundary. Our analysis shows that these parameters can be reliably obtained by using the mean resonance spacing and decay rate of the long-living resonances. Compared to the assumption of complete wave chaos, the effective parameters are renormalized in a uniform way. This renormalization finds its natural explanation in the formation of short-lived reso- nances that resemble bouncing-ball modes, which changes the mean resonance spacing of long-living resonances [33], and the resonance trapping phenomenon [38], which changes the mean decay rate.

\section{Acknowledgments}

We would like to thank H. J. Stöckmann for discussions. Financial support from the DFG research group 760 and the European Commission via the Marie Curie Excellence grant MEXT-2003-02778 is gratefully acknowledged.
[1] K. J. Vahala, Nature (London) 424, 839 (2003).

[2] P. Michler, A. Imamoğlu, M. D. Mason, P. J. Carson, G. F. Strouse, and S. K. Buratto, Nature (London) 406, 968 (2000).

[3] H.-G. Park, S.-H. Kim, S.-H. Kwon, Y.-G. Ju, J.-K. Yang, J.-H. Baek, S.-B. Kim, and Y.-H. Lee, Science 305, 1444 (2004).

[4] S. M. Ulrich, C. Gies, S. Ates, J. Wiersig, S. Reitzenstein, C. Hofmann, A. Löffler, A. Forchel, F. Jahnke, and P. Michler, Phys. Rev. Lett. 98, 043906 (2007).

[5] J. Reithmaier, G. Sek, A. Löffler, C. Hofmann, S. Kuhn, S. Reitzenstein, L. Keldysh, V. Kulakovskii, T. Reinecke, and A. Forchel, Nature (London) 432, 197 (2004).

[6] E. Peter, P. Senellart, D. Martrou, A. Lemaitre, J. Hours, J. M. Gérard, and J. Bloch, Phys. Rev. Lett. 95, 067401 (2005).

[7] M. Schwab, H. Kurtze, T. Auer, T. Berstermann, M. Bayer, J. Wiersig, N. Baer, C. Gies, F. Jahnke, J. Reithmaier, et al., Phys. Rev. B 74, 045323 (2006).

[8] J. U. Nöckel and A. D. Stone, Nature (London) 385, 45 (1997).

[9] C. Gmachl, F. Capasso, E. E. Narimanov, J. U. Nöckel, A. D. Stone, J. Faist, D. L. Sivco, and A. Y. Cho, Science 280, 1556 (1998).

[10] G. D. Chern, H. E. Tureci, A. D. Stone, R. K. Chang, M. Kneissl, and N. M. Johnson, Appl. Phys. Lett. 83, 1710 (2003).

[11] J. Wiersig and M. Hentschel, Phys. Rev. A 73, 031802(R) (2006).

[12] J. Wiersig and M. Hentschel, Phys. Rev. Lett. 100, 033901 (2008).

[13] Q. H. Song, H. Cao, B. Y. Liu, S. T. Ho, W. Fang and G. S. Solomon, arXiv:0810.3923 (2008).

[14] J.-W. Ryu, S. Rim, Y.-J. Park, C.-M. Kim, and S.-Y. Lee, Phys. Lett. A 372, 3531 (2008)

[15] O. A. Starykh, P. R. J. Jacquod, E. E. Narimanov, and A. D. Stone, Phys. Rev. E 62, 2078 (2000).

[16] M. Patra, Phys. Rev. E 67, 016603 (2003).

[17] G. Hackenbroich, J. Phys. A: Math. Gen. 38, 10537 (2005).

[18] Y. V. Fyodorov and H.-J. Sommers, J. Math. Phys. 38, 1918 (1997).

[19] T. S. Misirpashaev and C. W. J. Beenakker, Phys. Rev. A 57, 2041 (1998).

[20] H. J. Sommers, Y. V. Fyodorov, and M. Titov, J. Phys. A 32, L77 (1999).

[21] M. Patra, H. Schomerus, and C. W. J. Beenakker, Phys. Rev. A 61, 023810 (2000).

[22] K. M. Frahm, H. Schomerus, M. Patra, and C. W. J. Beenakker,
Europhys. Lett. 49, 48 (2000).

[23] H. Schomerus, K. M. Frahm, M. Patra, and C. W. J. Beenakker, Physica A 278, 469 (2000).

[24] U. Kuhl, R. Höhmann, J. Main, and H.-J. Stöckmann, Phys. Rev. Lett. 100, 254101 (2008).

[25] H. P. Baltes and E. R. Hilf, Spectra of Finite Systems (B-I Wissenschaftsverlag, Mannheim, 1978).

[26] T. Guhr, A. Müller-Groeling, and H. A. Weidenmüller, Phys. Rep. 299, 189 (1998).

[27] I. L. Aleiner and A. I. Larkin, Phys. Rev. B 54, 14423 (1996)

[28] P. Jacquod, H. Schomerus, and C. W. J. Beenakker, Phys. Rev. Lett. 90, 207004 (2003); J. Tworzydlo, A. Tajic, H. Schomerus, and C. W. J. Beenakker, Phys. Rev. B 68, 115313 (2003); H. Schomerus and Ph. Jacquod, J. Phys. A: Math. Gen. 38, 1066310682 (2005).

[29] S. Rahav and P. W. Brouwer, Phys. Rev. Lett. 95, 056806 (2005); S. Rahav and P. W. Brouwer, Phys. Rev. B 73, 035324 (2006); P. W. Brouwer and S. Rahav, Phys. Rev. B 74, 075322 (2006); P. W. Brouwer, Phys. Rev. B 76, 165313 (2007).

[30] P. Jacquod and R. S. Whitney Phys. Rev. B 73, 195115 (2006); R. S. Whitney and P. Jacquod, Phys. Rev. Lett. 96, 206804 (2006).

[31] J. Sjöstrand, Duke Mathematical Journal 60, 1 (1990).

[32] W. T. Lu, S. Sridhar, and M. Zworski, Phys. Rev. Lett. 91, 154101 (2003).

[33] H. Schomerus and J. Tworzydło, Phys. Rev. Lett. 93, 154102 (2004).

[34] S. Nonnenmacher and M. Zworski, J. Phys. A: Math. Gen. 38, 10683 (2005).

[35] J. P. Keating, M. Novaes, S. D. Prado, and M. Sieber, Phys. Rev. Lett. 97, 150406 (2006).

[36] J. Wiersig and J. Main, Phys. Rev. E 77, 036205 (2008).

[37] J. P. Keating, M. Novaes, and H. Schomerus, Phys. Rev. A 77, 013834 (2008).

[38] J. Okolowicz, M. Ploszajczak, and I. Rotter, Phys. Rep. 374, 271 (2003).

[39] S. W. McDonald and A. N. Kaufman, Phys. Rev. A 37, 3067 (1988).

[40] M. V. Berry, Eur. J. Phys. 2, 91 (1981).

[41] M. Robnik, J. Phys. A 16, 3971 (1983).

[42] J. Nagler, M. Krieger, M. Linke, J. Schönke, and J. Wiersig, Phys. Rev. E 75, 046204 (2007).

[43] J. D. Jackson, Classical Electrodynamics (John Wiley and Sons, New York, 1962). 
[44] M. R. Zirnbauer, J. Math. Phys. 37, 4986 (1996); A. Altland and M. R. Zirnbauer, Phys. Rev. B 55, 1142 (1997).

[45] The definition of $p_{l}$ in Eq. (10) of Ref. [37] contains a misprint. In the continuum limit $M \rightarrow \infty, p$ is supposed to cover the range $(-1,1)$.

[46] K. Zyczkowski and H.-J. Sommers, J. Phys. A: Math. Gen. 33, $2045(2000)$.

[47] Y. V. Fyodorov and H.-J. Sommers, JETP Lett. 72, 422 (2000); Y. V. Fyodorov and H.-J. Sommers, J. Phys. A: Math. Gen. 36, 3303 (2003)

[48] Y. Wei and Y. V. Fyodorov, J. Phys. A: Math. Theor. 41, 502001 (2008).

[49] S. Nonnenmacher and E. Schenck, Phys. Rev. E 78, 045202(R) (2008).

[50] L. A. Bunimovich, Funct. Anal. Appl. 8, 254 (1974).

[51] L. A. Bunimovich, Commun. Math. Phys. 65, 295 (1979).

[52] G. Benettin and J.-M. Strelcyn, Phys. Rev. A 17, 773 (1978).

[53] S. Shinohara, T. Harayama, H. E. Tureci, and A. D. Stone, Phys. Rev. A 74, 033820 (2006).
[54] T. Fukushima, T. Harayama, and J. Wiersig, Phys. Rev. A 73, 023816 (2006).

[55] M. Lebental, J. S. Lauret, J. Zyss, C. Schmit, and E. Bogomolny, Phys. Rev. A 75, 033806 (2007).

[56] S. Shinohara and T. Harayama, Phys. Rev. E 75, 036216 (2007).

[57] S. Shinohara, T. Fukushima, and T. Harayama, Phys. Rev. A 77, 033807 (2008).

[58] J. Wiersig, J. Opt. A: Pure Appl. Opt. 5, 53 (2003).

[59] J. Main, Phys. Rep. 316, 233 (1999).

[60] A. Richter, in Emerging Applications of Number Theory, The IMA Volumes in Mathematics and its Applications 109, 479 (Springer, New York, 1999).

[61] P. G. Silvestrov, M. C. Goorden, and C. W. J. Beenakker, Phys. Rev. Lett. 90, 116801 (2003).

[62] P. Jacquod and E.V. Sukhorukov, Phys. Rev. Lett. 92, 116801 (2004).

[63] P. W. Brouwer and S. Rahav, Phys. Rev. B 74, 085313 (2006). 